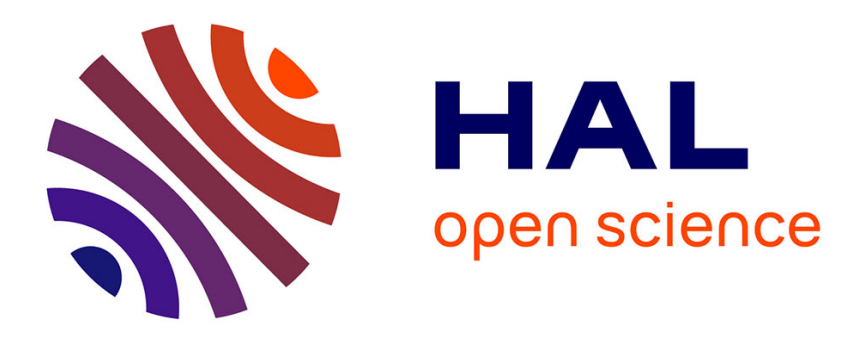

\title{
Vision-based self-calibration and control of parallel kinematic mechanisms without proprioceptive sensing
}

Nicolas Andreff, Philippe Martinet

\section{To cite this version:}

Nicolas Andreff, Philippe Martinet. Vision-based self-calibration and control of parallel kinematic mechanisms without proprioceptive sensing. Intelligent Service Robotics, 2009, 2 (2), pp.71-80. hal00520173

\section{HAL Id: hal-00520173 \\ https://hal.science/hal-00520173}

Submitted on 22 Sep 2010

HAL is a multi-disciplinary open access archive for the deposit and dissemination of scientific research documents, whether they are published or not. The documents may come from teaching and research institutions in France or abroad, or from public or private research centers.
L'archive ouverte pluridisciplinaire HAL, est destinée au dépôt et à la diffusion de documents scientifiques de niveau recherche, publiés ou non, émanant des établissements d'enseignement et de recherche français ou étrangers, des laboratoires publics ou privés. 


\title{
Vision-based Self-Calibration and Control of Parallel Kinematic Mechanisms without Proprioceptive Sensing
}

Received: date / Accepted: date

\begin{abstract}
This work is a synthesis of our experience over parallel kinematic machine control, which aims at changing the standard conceptual approach to this problem. Indeed, since the task space, the state space and the measurement space can coincide in this class of mechanisms, we came to redefine the complete modeling, identification and control methodology. Thus, it is shown in this paper that, generically and with the help of sensor-based control, this methodology does not require any joint measurement, thus opening a path to simplified mechanical design and reducing the number of kinematic parameters to identify. This novel approach is illustrated on the reference parallel kinematic mechanism (the Gough-Stewart platform) with vision as the exteroceptive sensor.
\end{abstract}

Keywords Parallel robots · Visual servoing · Robot calibration · Control

\section{Introduction}

There is a huge amount of literature devoted to parallel kinematic machines, which we will not reference here, except for Merlet (2000) which is a good

This work was supported by European Community through the Integrated Project NEXT number 0011815. It was initially published in the Proceedings of the 13th International Conference on Advanced Robotics (ICAR'07), Jeju, Korea, August 21-24, 2007. During this work, Philippe Martinet was holding a visiting professor position at the Intelligent Service Robotics Center at Sungkyunkwan University, Suwon, South Korea.

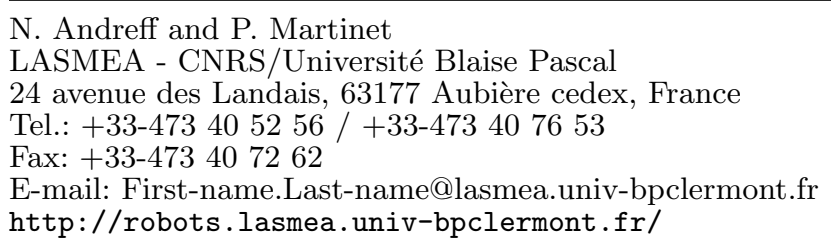


starting point in the area. However, to our knowledge, only little work is devoted to control and among these, none has addressed the problem of their control having in mind the serial/parallel duality exhibited by Waldron and Hunt (1991). Instead, as far as we know, all published work applies directly the standard control techniques coming from serial robotics to parallel robotics.

Consequently, in this introduction, we mainly want to remind the differences between serial and parallel kinematic mechanisms, then to point out the fundamental consequence thereof concerning control, which is the starting point of the present work.

The end-effector pose $X$ of a serial kinematic mechanism can be expressed in closed form from the joint values $\mathbf{q}$ using the so-called forward kinematic model:

$$
X=F K M(\mathbf{q}, \xi)
$$

where $\xi$ is the set of kinematic parameters. The actual expression of this relation may vary according to the representation which is chosen for the end-effector pose $X$.

From this expression, one can obtain the differential forward kinematic model, expressing the end-effector Cartesian velocity from the joint velocities, through formal time derivation:

$$
\tau=\mathbf{D}(\mathbf{q}, \xi) \dot{\mathbf{q}}
$$

where $\tau$ is expressed at any convenient point and in any reference frame, but usually at the end-effector frame origin and either in the base or end-effector frame (i.e. ${ }^{b} \tau_{e}$ or ${ }^{e} \tau_{e}$ ).

Thus, for serial kinematic mechanisms, the models depend only on the joint values. Consequently, the state of a serial kinematic mechanism is the joint value vector.

On the other hand, most parallel kinematic mechanisms have an inverse kinematic model, giving a closed-form expression of the relation from the end-effector pose to the joint values:

$$
\mathbf{q}=\operatorname{IKM}(X, \xi)
$$

Time differentiating (3), one can similarly obtain the differential inverse kinematic model, expressing the joint velocities from the end-effector Cartesian velocity:

$$
\dot{\mathbf{q}}=\mathbf{D}^{i n v}(X, \xi) \tau
$$

Thus, for parallel kinematic mechanisms, the models depend on the endeffector pose. Consequently, the state of a parallel kinematic mechanism is any representation of the end-effector pose $X$.

Notice, once again, that the differential inverse kinematic model, which is the heart of Cartesian control, has a closed-form expression for parallel kinematic mechanisms while it has to be numerically evaluated for serial kinematic mechanisms. Consequently, it should be more natural to perform Cartesian control for parallel kinematic mechanisms than for serial ones, provided that one has a correct estimate or measure of the end-effector pose. This was shown earlier in Dallej et al (2006) but, to be sure that a joint-free 


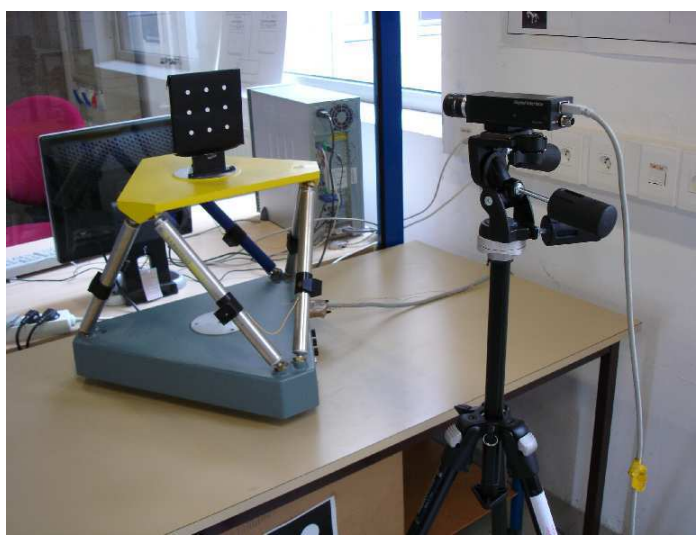

Fig. 1 A Gough-Stewart platform observed by a camera.

control is feasible, one has to check that the kinematic parameters used for that control can also be obtained without joint sensing.

The purpose of this paper is therefore to show that modeling, identification and control of parallel kinematic mechanisms can be derived without making any use of the joint values, since their state space coincides with the task space. In fact, in the parallel case, the three spaces (task, state and measurement spaces) needed for controling a robot can coincide, which is impossible in the serial case. Indeed, there exist several technologies for exteroceptive sensing, that is to perform measurement directly in the task space, among which the most versatile is computer vision.

As stated above, this paper is essentially a synthesis of our experience on kinematic control of parallel kinematic mechanisms and is based on previous work. Namely, the work in Dallej et al (2006) dealing with control is reformulated in the common joint sensor-free framework, while the joint sensor-free identification part is, if not of a high novelty, newly elaborated from the litterature state-of-the-art to give the missing element in a complete joint sensor-free use of parallel kinematic mechanisms. Indeed, the work in Dallej et al (2006) did not address at all whether joint sensor-free control could be performed without needing joint sensing at calibration time or not.

This paper is hence organized as follows. Section II addresses the kinematic modeling of parallel kinematic mechanisms when an exteroceptive sensor is a priori chosen to be used for control. Notice that this a priori choice is not usual in robotics since usually models are derived independently from which control (joint, model-based or sensor-based) will be implemented finally. Then, Section III is devoted to the most straightforward control which can be derived from the models in Section II and Section IV shows that calibration can also be performed without using any joint reading. Section V checks the validity of the approach on the much studied Gough-Stewart platform (Figure 1 and Gough and Whitehall (1962); Stewart (1965)). Finally, the paper ends on a discussion in Section VI. 


\section{Exteroceptive sensor-based kinematic modeling}

When deriving the kinematic model of any mechanism, the representation of the end-effector pose $X$ is usually expressed at the origin of the endeffector frame $\mathcal{F}_{e}$ and in the base frame $\mathcal{F}_{b}$. If one uses a homogeneous matrix representation of $X$, this means that one expresses the inverse kinematic model as a function of ${ }^{b} \mathbf{T}_{e}$.

However, both the base frame and the end-effector frame are arbitrary. Usually, they are chosen such that they can be easily interpreted by a human being and especially with regards to the task to be performed. Most often, this means a materialisation associated to the base on the one hand and to the end-effector on the other hand.

For instance, for a $6 \mathrm{R}$ serial kinematic mechanism, the base frame is usually located at the origin of the first joint and oriented such that it fits with the second body frame when the first joint is in its zero position. As for the end-effector frame, it is located at the center of the wrist.

However, if this is easy to represent physically the reference frames (although it remains a convenient approximation: who can say where precisely is the center of a wrist ?), it is not that easy for a human being to estimate the end-effector pose with respect to the base frame. Therefore, another convenient way of choosing a base frame, which is not related anymore to the physical base of the robot, is known as the zero-reference point method (see for instance Ruf and Horaud (1999)): the base frame and the end-effector frames coincide in a given reference configuration of the robot. In this case, it becomes more intuitive to program the robot trajectory since the latter is now a relative path rather than an absolute one.

Thus, the humanly interpretable choice is not necessary for control and it might be wise to choose the frames such that control and calibration are simplified. In fact, there is more freedom in the choice of the base frame than in the choice of the end-effector frame, since the latter must be coherent with the task to be performed.

In the case where an exteroceptive sensor is used (laser+retroflective cube, camera+pattern, ...), the base frame and the reference frame can generically be chosen as the sensor reference frame $\mathcal{F}_{r}$ and the sensor target frame $\mathcal{F}_{t}$ (or vice-versa), depending on whether the sensor is mounted on the mobile platform (eye-in-hand configuration, Fig. 2) or attached to the base (eye-tohand configuration, Fig. 3). To deal generically with the two configurations, let us introduce a sensor mobile frame $\mathcal{F}_{m}$ (which is the moving frame of the sensor+target couple) and a sensor fixed frame $\mathcal{F}_{f}$ (Figure 4 ).

Notice also that this systematic choice implies to calibrate the robot, since now, the physical measurements are not enough.

Consequently, in a totally generic manner, provided nevertheless that it exists, the inverse kinematic model can be expressed as a function of the pose of the sensor mobile frame $\mathcal{F}_{m}$ with respect to the sensor fixed frame $\mathcal{F}_{f}$ and of the kinematic parameters, expressed with respect to the latter frames, $\xi_{f, m}$ :

$$
\mathbf{q}=I K M\left({ }^{f} \mathbf{T}_{m}, \xi_{f, m}\right)
$$




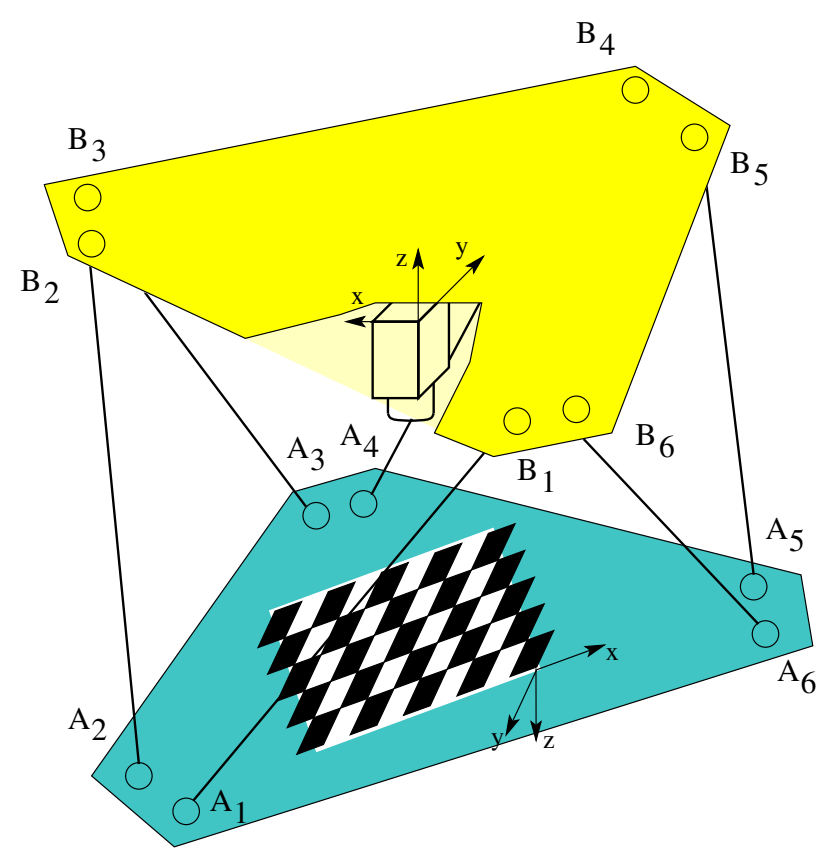

Fig. 2 A Gough-Stewart platform in eye-in-hand configuration, where a camera is attached to the mobile platform and observes a pattern tied to the base.

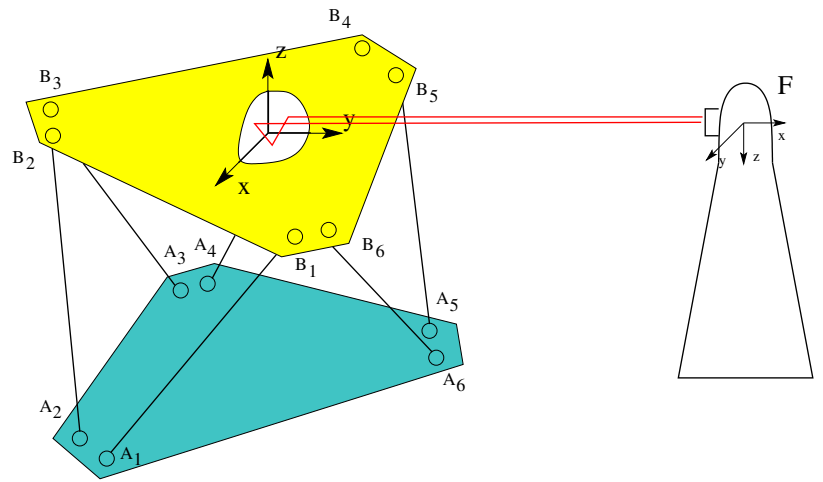

Fig. 3 A Gough-Stewart platform in eye-to-hand configuration, where a retroreflective cube is attached to the mobile platform and is tracked by a laser tracker fixed with respect to the base.

Thus, the joint sensors deliver an indirect observation of the state of a parallel kinematic mechanism, instead of a direct one in the serial case.

Notice that, most often, the above expression can be splitted in independent expressions for each leg:

$$
\forall i=1 . . n, q_{i}=I K M_{i}\left({ }^{f} \mathbf{T}_{m}, \xi_{i, f, m}\right)
$$




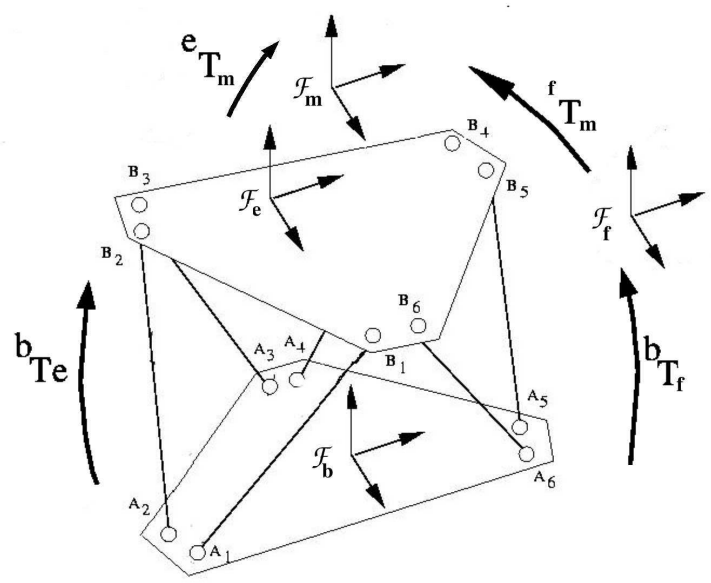

Fig. 4 Generic configuration for parallel kinematic mechanisms

exhibiting a specific subset $\xi_{i, f, m}$ of the kinematic parameters for each leg, without intersection with the subsets associated to the other legs.

\section{Example}

For instance, the kinematics of the well-known Delta robot presented in Clavel (1991) is usually obtained by writing the so-called implicit kinematic model as:

$$
\forall i=1 . .3, L_{i}^{2}=\left\|{ }^{b} \mathbf{R}_{e}{ }^{e} \mathbf{B}_{i}+{ }^{b} \mathbf{t}_{e}-{ }^{b} \mathbf{A}_{i}-l_{i} \cos q_{i}{ }^{b} \underline{\mathbf{x}}_{i}-l_{i} \sin q_{i}{ }^{b} \underline{\mathbf{y}}_{i}\right\|^{2}
$$

where $\mathbf{A}_{i}$ is the position of the actuator of leg $i$ on the base, $\underline{\mathbf{x}}_{i}$ and $\underline{\mathbf{y}}_{i}$ are a basis in the rotation plane associated to actuator $i$, $\mathbf{B}_{i}$ is the attachment point of leg $i$ onto the mobile platform, $\left(L_{i}, l_{i}\right)$ are the forearm and arm lengths of the leg elements and $\left({ }^{b} \mathbf{R}_{e},{ }^{b} \mathbf{t}_{e}\right)$ are the rotation matrix and position vector associated to the rigid transformation ${ }^{b} \mathbf{T}_{e}$.

However, since the norm is invariant to Euclidean changes of frames, the implicit kinematic model becomes:

$$
\forall i=1 . .3, L_{i}^{2}=\left\|{ }^{f} \mathbf{R}_{m}{ }^{m} \mathbf{B}_{i}+{ }^{f} \mathbf{t}_{m}-{ }^{f} \mathbf{A}_{i}-l_{i} \cos q_{i}{ }^{f} \underline{\mathbf{x}}_{i}-l_{i} \sin q_{i}{ }^{f} \underline{\mathbf{y}}_{i}\right\|^{2}
$$

From this implicit kinematic model, one obtains the inverse kinematic model by solving a second order polynomial in $t=\tan \frac{q_{i}}{2}$ yielding an expression of the form given in (6) where the set of the kinematic parameters $\left(\xi_{i, f, m}=\left\{{ }^{m} \mathbf{B}_{i},{ }^{f} \mathbf{A}_{i},{ }^{f} \underline{\mathbf{x}}_{i},{ }^{f} \underline{\mathbf{y}}_{i}, L_{i}, l_{i}\right\}\right)$ is expressed relatively to the sensor fixed frame and the sensor mobile frame.

Now, the attachement points of the legs on the fixed base and the associated reference vectors are to be calibrated in the sensor fixed 
frame, while the attachment points on the mobile platform are to be calibrated in the sensor mobile frame.

A second consequence of the generic choice of the frames is that the differential inverse kinematic model of a parallel kinematic mechanism writes generically:

$$
\forall i, \dot{q}_{i}={ }^{m} \mathbf{D}_{m, i}^{i n v}\left({ }^{f} \mathbf{T}_{m}, \xi_{f, m}\right)^{m} \tau_{m}
$$

Let us now leave the Delta aside and come back to the generic case.

\section{Exteroceptive sensor-based control}

Since the state of a parallel kinematic mechanism is its end-effector pose (provided that the inverse kinematic model has a closed-form expression), it is straightforward to derive a non-linear state feedback control law. To do so, one just needs to consider the following signal, to be servoed to 0 :

$$
{ }^{m} \mathbf{S}=\left(\begin{array}{c}
m \\
{ }^{m} \mathbf{t}_{m^{*}} \\
{ }^{m} \underline{\mathbf{u}} \theta
\end{array}\right)
$$

where ${ }^{m} \mathbf{t}_{m^{*}}$ is the position error or translation between the current $\left(\mathcal{F}_{m}\right)$ and desired $\left(\mathcal{F}_{m^{*}}\right)$ sensor mobile frame, while ${ }^{m} \underline{\mathbf{u}} \theta$ is the orientation error, decomposed as the axis ${ }^{m} \underline{\mathbf{u}}$ and angle $\theta$ of the rotation ${ }^{m} \mathbf{R}_{m^{*}}$ between these two frames. Indeed, this signal is a representation of the end-effector pose since it is obtained from the rigid transformation ${ }^{m} \mathbf{T}_{m^{*}}$ obtained by composition of the end-effector pose, expressed in the sensor fixed frame and the sensor mobile frame, in the current $\left({ }^{f} \mathbf{T}_{m}\right)$ and desired $\left({ }^{f} \mathbf{T}_{m^{*}}\right)$ state. Thus, this control signal is a function of the end-effector pose:

$$
{ }^{m} \mathbf{s}=f\left({ }^{f} \mathbf{T}_{m}\right)
$$

and conversely, one has a closed-form expression for the end-effector pose given ${ }^{m} \mathbf{s}$ using the Rodrigues formula and basic composition of rigid transformations.

Notice that when this signal is reconstructed from image information, then one gets the so-called 3D pose visual servoing scheme (Thuilot et al (2002); Wilson et al (1996)) and that expressing this signal in the current sensor mobile frame $\mathcal{F}_{m}$ yields a complete decoupling of the translation and orientation (Thuilot et al (2002)).

The time derivative of the control signal ${ }^{m} \mathbf{s}$ is given by:

$$
\frac{d^{m} \mathbf{s}}{d t}=\mathbf{L}\left({ }^{m} \mathbf{s}\right){ }^{m} \tau_{m}
$$

where all one needs to know in this paper about the interaction matrix $\mathbf{L}\left({ }^{m} \mathbf{s}\right)$ is that it is square (Malis et al (1999); Thuilot et al (2002)) and can be formally inverted (Malis et al (1999)). Then, the pseudo-control input ensuring exponential convergence of ${ }^{m} \mathbf{s}$ is obtained by:

$$
{ }^{m} \tau_{m}=-\lambda \mathbf{L}^{-1}\left({ }^{m} \mathbf{s}\right)^{m} \mathbf{S} \quad \text { with } \quad \lambda>0
$$


However, from (11), one can rewrite the above pseudo-control as

$$
{ }^{m} \tau_{m}=-\lambda \mathbf{L}^{-1}\left(f\left({ }^{f} \mathbf{T}_{m}\right)\right){ }^{m} \mathbf{s}, \lambda>0
$$

which only depends on the end-effector pose.

Then, according to (9), this pseudo-control is casted into the actual joint velocity control signal:

$$
\forall i, \dot{q}_{i}=-\lambda^{m} \mathbf{D}_{m, i}^{i n v}\left({ }^{f} \mathbf{T}_{m}, \xi_{f, m}\right) \mathbf{L}^{-1}\left(f\left({ }^{f} \mathbf{T}_{m}\right){ }^{m} \mathbf{s}, \lambda>0\right.
$$

Let us underline that this control law is the same for both the eye-inhand and the eye-to-hand configurations, provided that ${ }^{f} \mathbf{T}_{m}$ is adequately obtained:

Eye-to-hand configuration: Here, the sensor mobile frame $\mathcal{F}_{m}$ is the sensor target frame $\mathcal{F}_{t}$ and the sensor fixed frame $\mathcal{F}_{f}$ is the sensor reference frame $\mathcal{F}_{r}$ and hence the transformation ${ }^{f} \mathbf{T}_{m}$ is directly delivered by the sensor:

$$
{ }^{f} \mathbf{T}_{m}={ }^{r} \mathbf{T}_{t}
$$

Eye-in-hand configuration: Here, the sensor mobile frame $\mathcal{F}_{m}$ is the sensor reference frame $\mathcal{F}_{r}$ (e.g. the camera frame) and the sensor fixed frame $\mathcal{F}_{f}$ is the sensor target frame $\mathcal{F}_{t}$ (e.g. the visual pattern frame) and hence the transformation ${ }^{f} \mathbf{T}_{m}$ is the inverse of the transformation delivered by the sensor:

$$
{ }^{f} \mathbf{T}_{m}={ }^{r} \mathbf{T}_{t}^{-1}
$$

The above control does not make any assumption on which exteroceptive sensor is used. It could thus as well be a vision system as a laser tracker or a GPS-like sensor or even a serial mechanism with joint encoders (provided yet that no force nor torque is transmitted in this redundant kinematic chain in the parallel kinematic mechanism). Our opinion is that vision should be a good balance between cost, versatility, accuracy and robustness to working conditions, but this would deserve a longer discussion taking into account all the industrial requirements and technological constraints.

In particular, the control law presented above is the most straightforward, but requires the observation of the pose, which is not an easy task. It relies indeed either on very accurate mechanical realisation (laser tracker, redundant mechanism) or on non-linear numerical solving (pose estimation by vision). Higher performances could, on the opposite, be achieved using control laws with other sensor signals as it will be discussed in the last section.

\section{Exteroceptive sensor-based self-calibration}

In summary of the last two sections, parallel kinematic mechanisms can be modelled and controlled without any call to joint values, provided that the kinematic parameters are expressed in the adequate frames. However, because the latter frames are not intuitively defined, this joint-free control imposes the system to be calibrated and can not simply use the CAD values 
of the robot (unless the CAD model includes the exteroceptive sensor). Calibration must also be done without measuring the joint values, otherwise joint-free control is almost useless.

There are several ways to identify the kinematic parameters without using the joint values. The purpose of this section is not to give the technical details of a given calibration method but rather to suggest a couple of calibration alternatives and show the feasibility of the methodology. Notice, before that, that in the case where an exteroceptive sensor-based control is used, calibration does not need be very accurate since kinematic parameters only appear, in the control law, in the differential model and not in the servoed error.

Since only the differential inverse kinematic model is required for control, the most straightforward method would be to record simultaneously all the variables in (9), namely the mobile frame pose with respect to the fixed frame ${ }^{f} \mathbf{T}_{m}$, the instantaneous mobile frame Cartesian velocity ${ }^{m} \tau_{m}$ and the associated joint velocities $\dot{\mathbf{q}}$, but this raises two drawbacks. The first one is that one needs to measure simultaneously and accurately both a $3 \mathrm{D}$ pose and a 3D velocity, although it is partially solved by Ait-Aider et al (2006). The second one is that joint velocity measurements are required, which we want to get rid of. A solution lies in applying in the same joint velocity to a given leg $i$ in two different configurations $j$ and $k$. Thus, one can express the $i$ th leg joint velocity in configuration $j$ from the differential inverse kinematic model:

$$
\dot{\mathbf{q}}_{i}={ }^{m} \mathbf{D}_{m, i}^{i n v}\left({ }^{f} \mathbf{T}_{m}^{j}, \xi_{f, m}\right)^{m} \tau_{m}^{j}
$$

Expressing similarly the same leg joint velocity in configuration $k$ yields:

$$
{ }^{m} \mathbf{D}_{m, i}^{i n v}\left({ }^{f} \mathbf{T}_{m}^{j}, \xi_{f, m}\right)^{m} \tau_{m}^{j}={ }^{m} \mathbf{D}_{m, i}^{i n v}\left({ }^{f} \mathbf{T}_{m}^{k}, \xi_{f, m}\right)^{m} \tau_{m}^{k}
$$

This constraint equation involves the unknown kinematic parameters $\xi_{f, m}$ as well as the measured 3D pose and 3D velocity in both configurations but does not depend on the joint measurement. As a consequence, one can now solve for this non linear system, for several configuration triplets $(i, j, k)$ satisfying the constraints, to calibrate the kinematic parameters. Nevertheless, this requires that the application of a given joint velocity is sufficiently repeatable.

A more classical approach could rely on the classical calibration method using the inverse kinematic model (Zhuang et al (1998)), although some parameters may vanish with time differentiation. The adaptation of classical calibration methods using joint values to a joint-free version is based on the same principle of assigning known values to a given joint $i$. It is more convenient than in the differential case since the joint limits are two convenient joint values, that can either be easily measured at hand or simply imposed repeatedly. Notice that the assignment of such joint values does not need be very accurate since calibration does not either need be very accurate. Thereby, if a rough estimate of the joint limits can be obtained, the usual exteroceptive sensor-based calibration (or its variation based on the implicit kinematic model proposed by Wampler et al (1995)) can be used:

$$
\forall i, \min _{\xi_{i, f, m}} \frac{1}{2} \sum_{j}\left\|q_{i}^{j}-I K M_{i}\left({ }^{f} \mathbf{T}_{m}^{j}, \xi_{f, m}\right)\right\|^{2}
$$


where one minimizes the errors between the robot roughly estimated joint values $q_{i}^{j}$ and those obtained from the inverse kinematic model for each configuration.

Alternately, one can use the same principle as in the differential case, that is constraining one given joint $i$ to the same joint limit while placing the end-effector in two different poses $j$ and $k$ :

$$
\forall i, \min _{\xi_{i, f, m}} \frac{1}{2} \sum_{j, k}\left\|I K M_{i}\left({ }^{f} \mathbf{T}_{m}^{k}, \xi_{f, m}\right)-I K M_{i}\left({ }^{f} \mathbf{T}_{m}^{j}, \xi_{f, m}\right)\right\|^{2}
$$

where one minimizes the errors between the output of the inverse kinematic model in configuration $j$ and the one in configuration $k$.

This approach is the dual to self-calibration methods in serial robotics (Khalil and Dombre (2002)) where one applies constraints to the end-effector in order to avoid measuring its pose. It is also a variant of the constrained calibration method proposed in Daney (1999), except that the end-effector pose is measured rather than estimated through the Forward Kinematic Problem, which removes much of the risk of numerical instability reported by Daney and Emiris (2001).

Which of the above suggested methods is to be used for a given real application depends largely on the actual set-up and can not be determined generically yet.

Nevertheless, no joint sensor is required in any of the above methods. Thus, the joint values can be removed both from control and from calibration and hence, the mechanical design of parallel kinematic mechanisms can be simplified. Since the same exteroceptive sensor is used for calibration and for control, calibration can be performed autonomously by the robot, and can even be done on-line during control, either to simply monitor the kinematic parameters or to cope with slow variation of the latter.

\section{Experimental validation}

In the previous derivation, we did not make any assumption on which parallel kinematic mechanism was to be controlled, i.e. on the expression of the inverse kinematic model. In this section, the approach is experimentally validated on a commercial Gough-Stewart platform in eye-to-hand configuration (Figure 1).

\subsection{Inverse kinematic model}

The Gough-Stewart mechanism has 6 legs of varying length $\mathbf{q}_{i}, i \in 1$...6, attached to the base by spherical or universal joints located in points $\mathbf{A}_{i}$ and to the moving platform (end-effector) by spherical joints located in points $\mathbf{B}_{i}$. The implicit kinematic model of such an hexapod expressed in the endeffector frame is

$$
\forall i \in 1 . .6, \quad \mathbf{q}_{i}^{2}=e \overrightarrow{\mathbf{A}}_{i} \mathbf{B}_{i}^{T} e \overrightarrow{\mathbf{A}_{i} \mathbf{B}_{i}}
$$


expressing that $q_{i}$ is the length of vector $\overrightarrow{\mathbf{A}_{i} \mathbf{B}_{i}}$. One can then trivially obtain the inverse kinematic model expression given by Merlet (2000).

The implicit kinematic model can be expressed in the sensor mobile frame, since the scalar product is invariant to any change of frames in the Euclidean space:

$$
\forall i \in 1 . .6, \quad \mathbf{q}_{i}^{2}={ }^{m}{\overrightarrow{\mathbf{A}_{i} \mathbf{B}_{i}}}^{m} \vec{m}_{\mathbf{A}_{i} \mathbf{B}_{i}}
$$

Introducing $\underline{\mathbf{u}}_{i}$ the unit vector pointing from $\mathbf{A}_{i}$ to $\mathbf{B}_{i}$, we can rewrite (23) as

$$
q_{i}{ }^{m} \underline{\mathbf{u}}_{i}={ }^{m} \mathbf{B}_{i}-{ }^{m} \mathbf{R}_{f}{ }^{f} \mathbf{A}_{i}-{ }^{m} \mathbf{t}_{f}
$$

from which one obtains the differential inverse kinematic model

$$
\dot{\mathbf{q}}={ }^{m} \mathbf{D}_{m}^{i n v}{ }^{m} \tau_{m}
$$

with

$$
{ }^{m} \mathbf{D}_{m}^{i n v}=\left(\begin{array}{ccc}
{ }^{m} \underline{\mathbf{u}}_{1}^{T} & { }^{m} \mathbf{B}_{1} \times{ }^{m} \underline{\mathbf{u}}_{1}^{T} \\
\vdots & \vdots & \\
{ }^{m} \underline{\mathbf{u}}_{6}^{T} & { }^{m} \mathbf{B}_{6} \times{ }^{m} \underline{\mathbf{u}}_{6}^{T}
\end{array}\right)
$$

and

$$
{ }^{m} \underline{\mathbf{u}}_{i}=\frac{{ }^{m} \mathbf{B}_{i}-{ }^{m} \mathbf{R}_{f}{ }^{f} \mathbf{A}_{i}-{ }^{m} \mathbf{t}_{f}}{\left\|{ }^{m} \mathbf{B}_{i}-{ }^{m} \mathbf{R}_{f}{ }^{f} \mathbf{A}_{i}-{ }^{m} \mathbf{t}_{f}\right\|}
$$

In the above expressions, the ${ }^{f} \mathbf{A}_{i}$ and the ${ }^{m} \mathbf{B}_{i}$ are the constant kinematic parameters to be calibrated. Notice that the joint offsets, a major source of modeling errors, disappeared from the set of calibration parameters.

\subsection{Calibration}

To calibrate the robot, we used the $2^{6}=64$ configurations where each leg is either stretched out or tucked in. First, we used these configurations to calibrate the robot using the joint sensor information. Thus, inserting the joint offsets in the model in (23) and developping the expression of vector $\overrightarrow{\mathbf{A}_{i} \mathbf{B}_{i}}$ as in (24) yields the detailed kinematic model:

$$
\forall i=1 . .6,\left(q_{i}+q_{0 i}\right)^{2}-\left\|{ }^{f} \mathbf{R}_{m}^{j}{ }^{m} \mathbf{B}_{i}+{ }^{f} \mathbf{t}_{m}^{j}-{ }^{f} \mathbf{A}_{i}\right\|^{2}=0
$$

and, consequently, the following standard minimization problem:

$$
\forall i, \min _{\xi_{i, f, m}} \frac{1}{2} \sum_{j=1}^{64}\left\|\left(q_{i}+q_{0 i}\right)^{2}-\right\|{ }^{f} \mathbf{R}_{m}^{j}{ }^{m} \mathbf{B}_{i}+{ }^{f} \mathbf{t}_{m}^{j}-{ }^{f} \mathbf{A}_{i}\left\|^{2}\right\|^{2}
$$

where the kinematic parameter set $\xi_{i, f, m}$ is composed of the joint offset $q_{0 i}$, the joint location on the base ${ }^{f} \mathbf{A}_{i}$ (expressed in the fixed frame) and the joint location on the mobile platform ${ }^{m} \mathbf{B}_{i}$ (expressed in the mobile frame). 

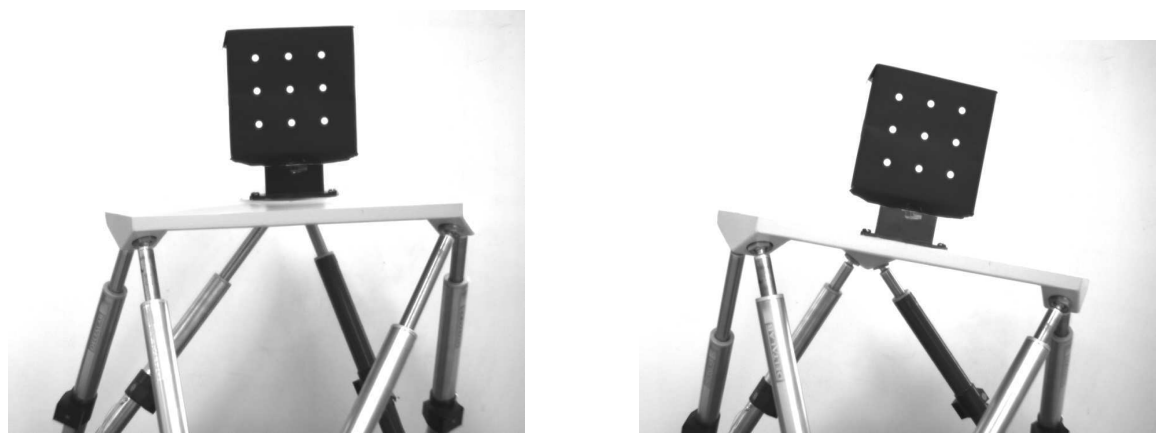

Fig. 5 Initial (left) and desired (right) position of the end-effector, seen from the camera.

Then, we calibrated the robot using the last method proposed above, consisting in using, for each leg, the $32 * 31=992$ pairs of the above configurations with the leg in the same joint limit:

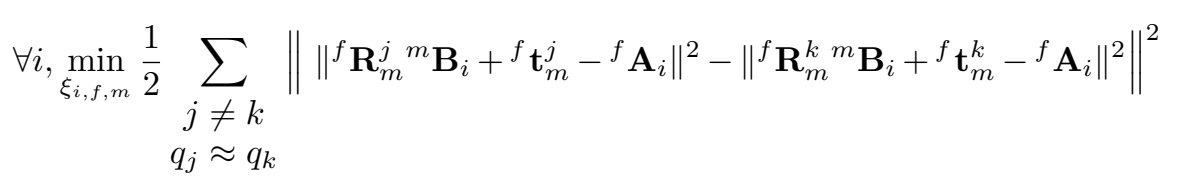

where one minimizes the error between the squared leg length (obtained from the inverse kinematic model) in pairs of configurations where they are physically the same. Notice that the joint offset disappeared and that the only unknowns in this problem are the joint locations ${ }^{f} \mathbf{A}_{i}$ and ${ }^{m} \mathbf{B}_{i}$.

Both calibration methods give results ${ }^{1}$ with a maximal deviation between the two methods of less than $0.3 \mathrm{~mm}$ on the positions of ${ }^{m} \mathbf{B}_{i}$ and ${ }^{f} \mathbf{A}_{i}$. Thus, as far as our control is concerned, calibrating without the joint values is equivalent, in practice, to calibrating with the joint values. Therefore, the theoretical opportunity of releasing the need for joint sensing is confirmed from a practical point of view.

\subsection{Control}

We present here some experimental results showing that control can be performed without any joint sensing either.

In the first reported experiment, the robot is asked to reach the desired position from the initial configuration that are displayed in Figure 5. Thus, the robot covers a large amount of its workspace.

Figure 6 not only shows that the errors converge to 0 as expected, from an initial error to a final one displayed in Table 1, but also that the motion in the Cartesian space is decoupled: the end-effector trajectory is a screw.

\footnotetext{
1 Notice that the exact value of the kinematic parameters before and after the calibration are not displayed here since they depend on the relative positioning of the sensing device with respect to the robot.
} 

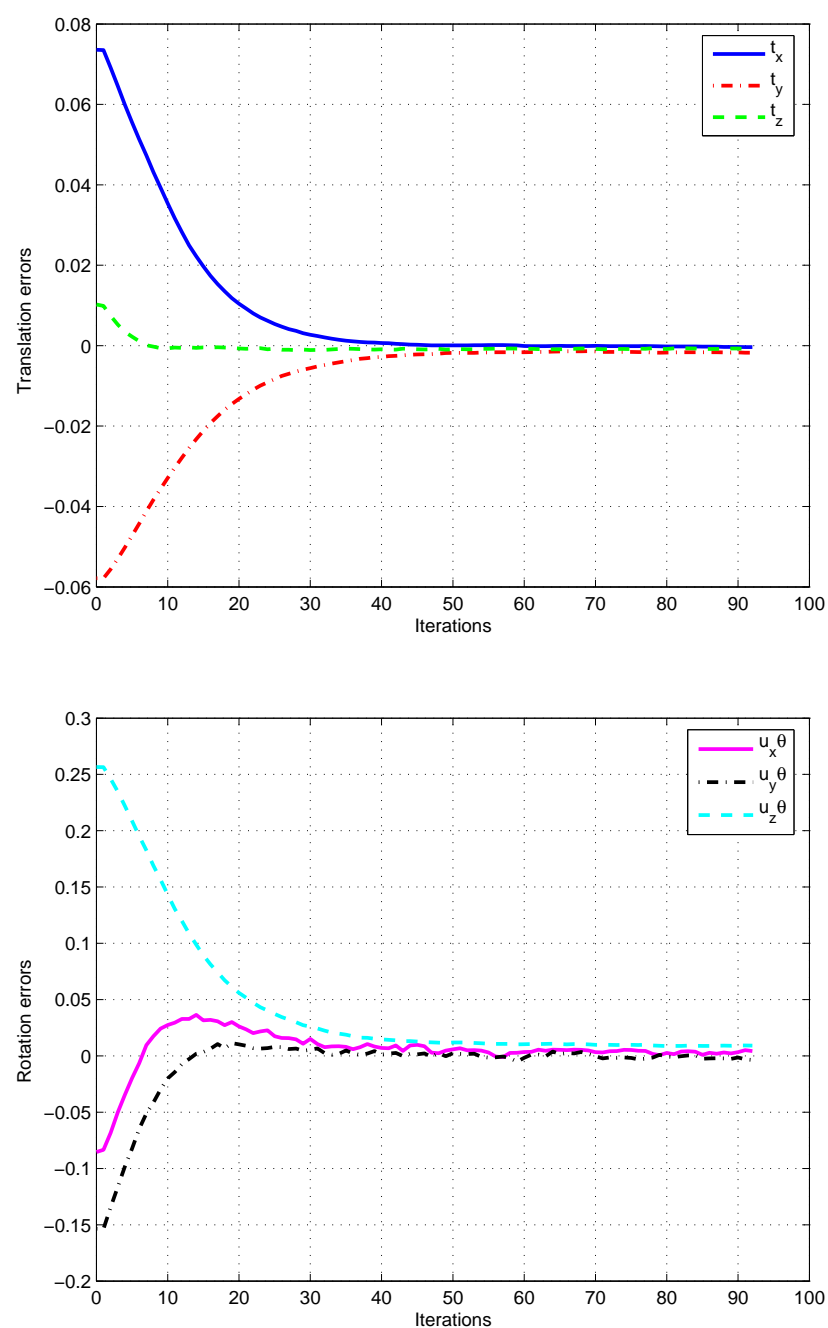

Fig. 6 Evolution of the translation error (top, in meters) and rotation error (bottom, in radians), with a control gain $\lambda=3$.

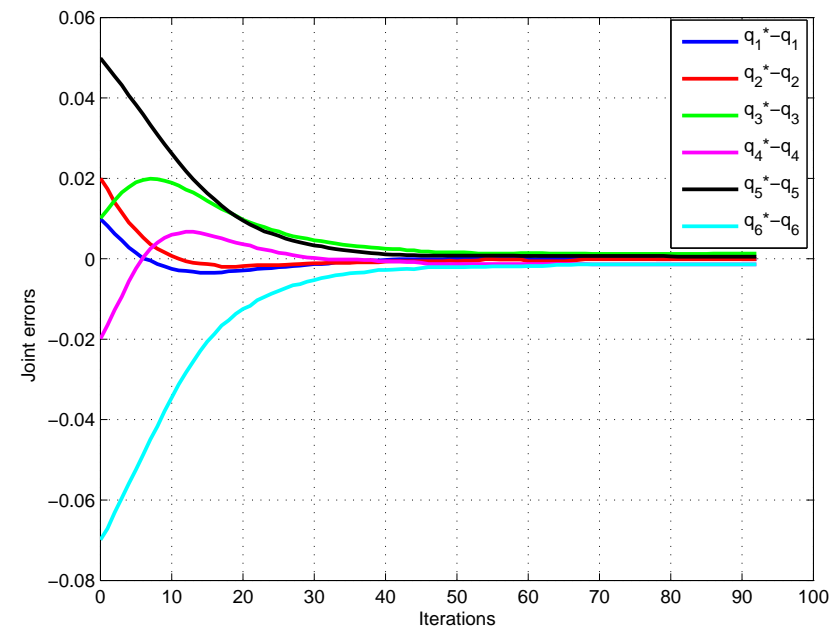

Fig. 7 Evolution of the joint errors (in meters), with a control gain $\lambda=3$. 
Table 1 Initial and final errors

\begin{tabular}{|l|c|c|}
\hline & Position error $(\mathrm{m})$ & Orientation error (rad) \\
\hline Initial Errors & 0.0942 & 0.312 \\
\hline Final Errors & 0.0017 & 0.008 \\
\hline
\end{tabular}

Since the platform is equipped with joint sensors, they were recorded during the control, although not used for the control itself. Figure 7 shows that convergence is also normally reached in the joint space. It also shows the joints coupled behaviour, associated to the fact that the control is decoupled in the Cartesian space.

Note that the effective accuracy of the convergence depends essentially on the pose estimation accuracy: under perfect rigid-body, backlash- and friction-free assumptions, this accuracy is in fact theoretically only dependent on the latter. It is, here, rather low (in the order of $0.5 \mathrm{~mm}$ and $0.001 \mathrm{rad}$ ) since only 9 points were used on the visual pattern, but the pose estimation could easily be improved to micrometric accuracy with state-of-the-art know-how, but this is not needed here.

Indeed, the effective accuracy may be limited by poor mechanical properties of the platform. Our actual set-up is thus impaired by backlashes in the spherical joints and high friction in the actuators.

To compensate for friction, one can increase the control gain (either globally or adaptively). On the one hand, Figure 8 shows the evolution of the errors when using a control gain equal to 8 instead of 3, as above. Convergence accuracy is improved to $0.6 \mathrm{~mm}$ and $0.003 \mathrm{rad}$ (in the order of both the implemented pose estimation and the mechanical repeatability of our set-up), but to the cost of a little overshoot and the risk of tracking loss due to high initial velocities. On the other hand, Figure 9 shows the evolution of the errors when using an adaptive control gain $\left(\lambda=0.8+(8-0.8) e^{-200\left\|^{m} \mathbf{s}\right\|}\right)$. Convergence is also improved to $0.7 \mathrm{~mm}$ and $0.004 \mathrm{rad}$, but then the convergence is not exponential anymore. Figure 10 displays the resulting joint errors in both cases. It shows that the residual joint errors are in the order of a millimeter, that is the one which can be achieved in practice with a simple joint control on this set-up.

\section{Discussion}

To sum up, this paper has shown that modeling, identification and control of parallel kinematic mechanisms, having a closed-form expression of their inverse kinematic model (that are the vast majority of parallel kinematic mechanisms), can be performed without any joint reading but with an exteroceptive sensor measuring the end-effector pose. To do so, one needs to express the models (and the end-effector pose) in the reference frames associated to the exteroceptive sensor, which has for consequence that calibration also needs be performed without joint sensing.

Although the approach was applied once in a real experiment, which shows the feasibility of the methodology, the actual efficiency of this method- 

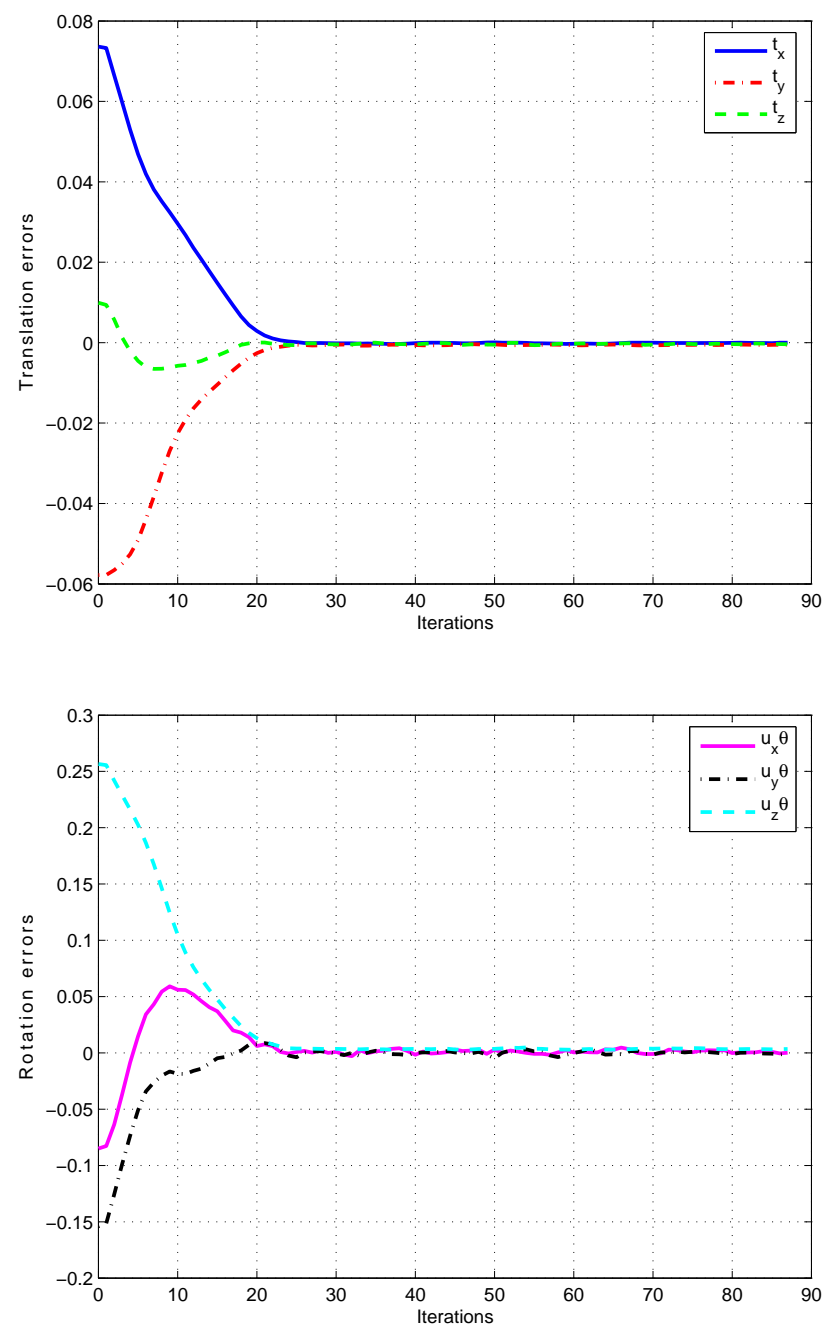

Fig. 8 Evolution of the translation error (top, in meters) and rotation error (bottom, in radians), with a control gain $\lambda=8$.

ology still deserves to be assessed through complete experimental validation on various manipulators.

Yet, it can already be foreseen that, using such an approach, mechanical design of parallel kinematic mechanisms can be simplified since fewer components need be assembled. Practical consequences of that should be higher repeatability and mechanical robustness as well as reduced friction and maintenance. Moreover, a parallel kinematic mechanism equipped with such an exteroceptive sensor should become able to monitor autonomously the parameters in its control model and, if necessary, perform self-calibration or 

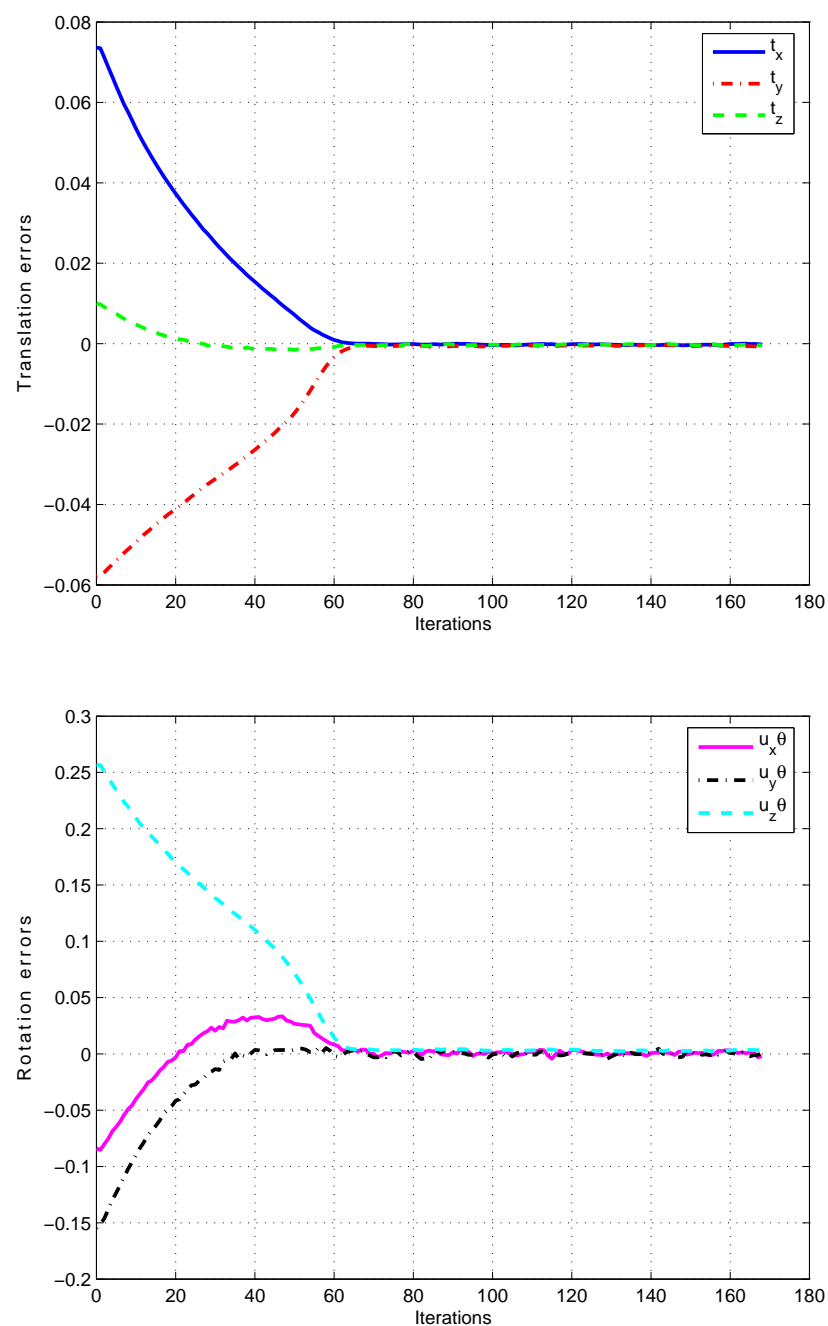

Fig. 9 Evolution of the translation error (top, in meters) and rotation error (bottom, in radians), with an adaptive control gain.

self-tuning control. Let us notice that these issues have recently been studied on a linear Delta equipped with an exteroceptive sensor consisting of a Gough platform Corbel (2008).

However, the methodology proposed in this paper requires to estimate the end-effector pose, either by inverting the simple camera projection model or by using complex systems (laser tracker or mechanical measuring device). Thus, the control signal might be biased through the estimation process, which would yield a bias in the final position even though the control converges. If the bias is constant over the whole workspace, this is not very 

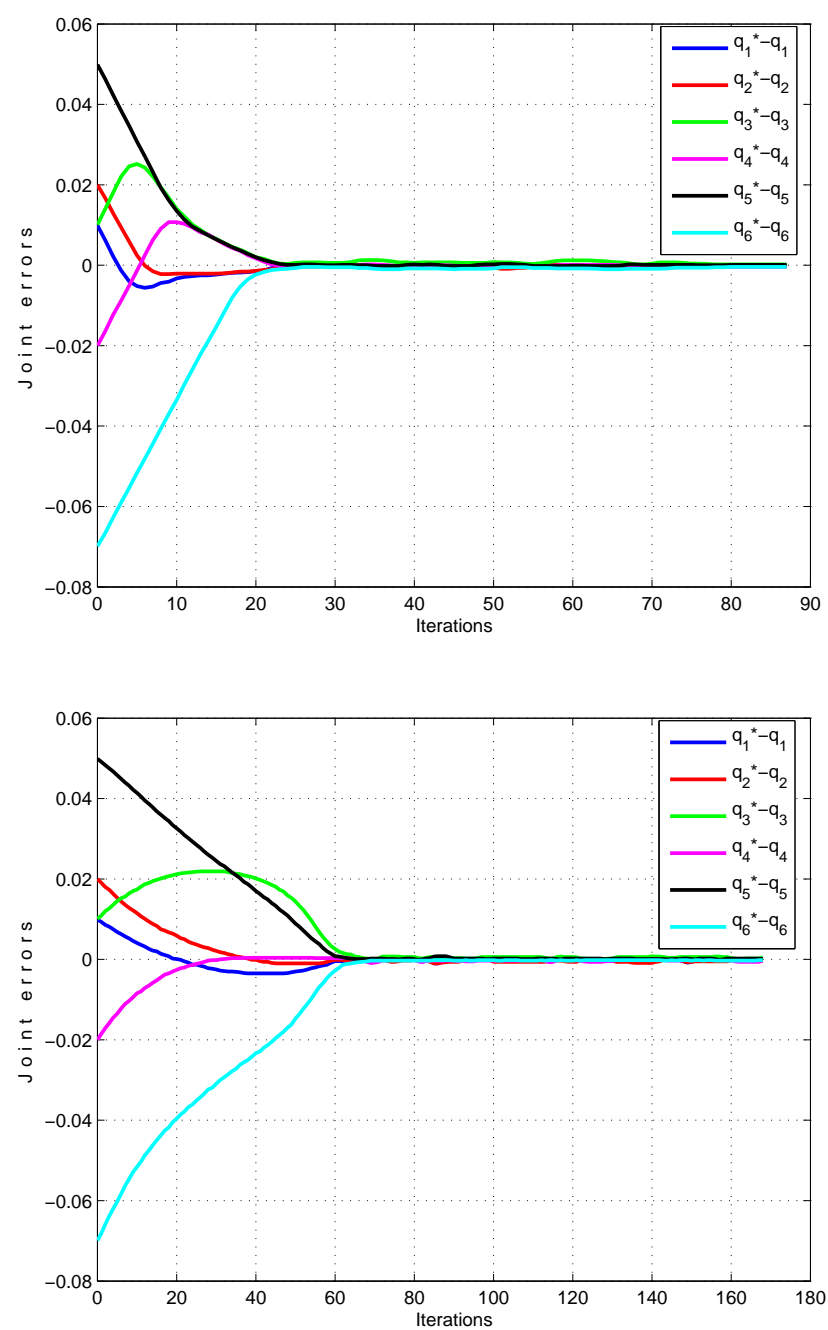

Fig. 10 Evolution of the joint errors (in meters) when $\lambda=8$ (top) and when using an adaptive gain (bottom) .

troublesome since it can be easily compensated for. However, if it varies over the workspace, then it might be necessary to head to control methods with a "cleaner" control signal, namely sensor-based control introduced in Samson et al (1991).

Consequently, one would use an indirect observation of the state of the parallel kinematic mechanism, where the control signal is an image of the state. This is actually what visual servoing (IBVS, HBVS and PBVS) is since the camera delivers an image which is function of the end-effector pose. Noticeably, it is also the case, in the context of parallel kinematic mechanisms, 
for joint control where joint values are a function of the end-effector pose. Thus, according to the serial/parallel duality, joint control becomes a sensorbased control.

However, the choice of the indirect observation shall be chosen in order that the estimation of the end-effector pose be simple. This discards somehow joint control because this would bring us back to the troublesome Forward Kinematic Problem. This also discards visual servoing methods where the interaction matrix is taken at convergence. Indeed, to avoid internal forces or undesired internal motions, the differential inverse kinematic model has to be estimated properly, and hence the end-effector pose has to be estimated at each control iteration.

A careful observation of the specific case of Gough-Stewart shows that the inverse kinematic matrix ${ }^{m} \mathbf{D}_{m}^{i n v}$ only depends on the direction of the legs. Thus, it is not necessary to estimate explicitly the end-effector pose, since a short-cut is possible by directly measuring the directions and using them as the control primitive as in Andreff and Martinet (2006). However, this solution still requires the reconstruction from the image of the direction, which can be noisy. Consequently, an image-based solution consisting of servoing the projection of the legs can be designed as in Andreff et al (2007) where the control is done directly in the sensor space and the 3D information can be easily reconstructed with simple algebra to feed the interaction matrix and the inverse kinematic matrix.

In conclusion, the next step to be made is to extend this search for the best indirect observation in a generic way for any parallel kinematic mechanism. The following step will then be to consider "pathological" parallel kinematic mechanisms that do not have a closed-form expression for the inverse kinematic model, such as the planar 3-RRR mechanism which can have several end-effector poses associated to a given joint configuration and several joint configurations associated to a given end-effector pose (Chablat and Wenger (1998)). A hint is to rely on the redundant metrology paradigm (Stoughton and Arai (1991); Merlet (1993); Baron and Angeles (2000); Marquet et al (2002)) not only to simplify the solution of the Forward Kinematic Model as it is used usually, but much preferably to derive a simple, yet performant, control.

\section{References}

Ait-Aider O, Andreff N, Lavest J, Martinet P (2006) Simultaneous object pose and velocity computation using a single view from a rolling shutter camera. In: Proceedings of the 9th European Conference on Computer Vision, ECCV'06, Graz, Austria, vol 2, pp 56-68

Andreff N, Martinet P (2006) Unifying kinematic modeling, identification and control of a Gough-Stewart parallel robot into a vision-based framework. IEEE Trans on Robotics 22(6):1077-1086

Andreff N, Dallej T, Martinet P (2007) Image-based visual servoing of a Gough-Stewart parallel manipulator using leg observations. International Journal of Robotics Research Special Issue on Vision and Robotics - Joint with the International Journal on Computer Vision 26(7):677-687 
Baron L, Angeles J (2000) The direct kinematics of parallel manipulators under joint-sensor redundancy. IEEE Trans on Robotics and Automation $16(1): 1-8$

Chablat D, Wenger P (1998) Working modes and aspects in fully-parallel manipulators. In: Proceedings of the 1998 IEEE Int. Conf. on Robotics and Automation, Leuven, Belgium, pp 1964-1969

Clavel R (1991) Conception d'un robot parallèle rapide à 4 degrés de liberté. PhD thesis, EPFL, Lausanne

Corbel D (2008) Contribution à l'amélioration de la précision des robots parallèles. Thèse de doctorat, Université Montpellier II

Dallej T, Andreff N, Martinet P (2006) 3D pose visual servoing is the canonical choice for the Cartesian control of parallel robots. In: Proceedings of the IEEE/RSJ Int. Conf. on Intelligent Robots and Systems, IROS'06, Beijing, China

Daney D (1999) Self calibration of Gough platform using leg mobility constraints. In: World Congress on the theory of machine and mechanisms, Oulu, Finland, pp 104-109

Daney D, Emiris I (2001) Variable elimination for reliable parallel robot calibration. In: 2nd Workshop on Computational Kinematics (CK2001), Korea, pp 133-144

Gough V, Whitehall S (1962) Universal tyre test machine. In: Proc. FISITA 9th Int. Tech. Congress, pp 117-137

Khalil W, Dombre E (2002) Modeling, Identification and Control of Robots. Taylor and Francis

Malis E, Chaumette F, Boudet S (1999) 2 1/2 d visual servoing. IEEE Tran On Robotics and Automation 12:238-250

Marquet F, Company O, Krut S, Pierrot F (2002) Enhancing parallel robots accuracy with redundant sensors. In: Proceedings of the 2002 Int. Conf. on Robotics and Automation, Washington, Washington DC

Merlet J (2000) Parallel robots. Kluwer Academic Publishers

Merlet JP (1993) Closed-form resolution of the direct kinematics of parallel manipulators using extra sensors data. In: IEEE Int. Conf. on Robotics and Automation (ICRA'93), Atlanta, pp 200-204

Ruf A, Horaud R (1999) Visual servoing of robot manipulators, part I : Projective kinematics. Int J on Robotics Research 18(11):1101 - 1118

Samson C, Le Borgne M, Espiau B (1991) Robot Control: the Task Function Approach. Clarendron Press, Oxford University Press, Oxford, UK

Stewart D (1965) A platform with six degrees of freedom. In: Proc. IMechE (London), vol 180, pp 371-386

Stoughton R, Arai T (1991) Optimal sensor placement for forward kinematics evaluation of a 6-dof parallel link manipulator. In: IEEE Int. Conf. on Intelligent Robots and Systems (IROS'91), Osaka, pp 785-790

Thuilot B, Martinet P, Cordesses L, Gallice J (2002) Position based visual servoing: keeping the object in the field of vision. Proceedings of the 2002 IEEE Int Conf on Robotics and Automation

Waldron K, Hunt K (1991) Series-parallel dualities in actively coordinated mechanisms. Int J on Robotics Research 10(5):473-480 
Wampler C, Hollerbach J, Arai T (1995) An implicit loop method for kinematic calibration and its application to closed-chain mechanisms. IEEE Trans on Robotics and Automation 11(5):710-724

Wilson W, Hulls C, Bell G (1996) Relative end-effector control using cartesian position-based visual servoing. IEEE Tran On Robotics and Automation 12(5):684-696

Zhuang H, Yan J, Masory O (1998) Calibration of Stewart platforms and other parallel manipulators by minimizing inverse kinematic residuals. J of Robotic Systems 15(7):395-405 